\title{
INDEX TO CHAPTERS 1 AND 2
}

A

Aberdeen, Marischal College, $21 n$

absolutism, 42

Acosta, Joseph de, 36

Adam, 2, 3

Aesop, 38

alchemy, $7 \&$ n, 18 \& n, 19, 22, 22n-23n

Alciatus, Andreas, 41

Alfraganus, 23n

algebra, 24

All Hallows, London, church of, 3n, 4

Alsted, Johann Heinrich, 21, 41

Encyclopaedia, 21

altar rails, 28

Amadis de Gaule, 37 \& n

America, 32n, 36, 41n

Ames, William, 28, 41

anatomy, 25

angels, $12 \mathrm{n}$

Angers, University of, $42 \mathrm{n}$

Antichrist, 3 \& n, 29, 32

antinomianism, 3, 29

Apianus, Petrus, 23n

Apollonius Pergaeus, 24n

Apologia remonstrantium, 28

Appian of Alexandria, 35

Arabic, 19, 38 \& n

Archimedes, 24n

architecture, 24

Ariosto, Lodovico, Orlando furioso, 38n

Aristarchus, 24n

Aristotelianism, 20, 20-21, 22, 30, 40 \& n, 41,43

Aristotle, 18, $20 \& \mathrm{n}, 21,25,32 \mathrm{n}, 40 \& \mathrm{n}, 41$, 43

De anima, 21

De coelo, 21

Ethica, 21, 40

Logica, 20

Metaphysica, 20, 21

Opera, 20

Physica, 20n, 21

Politica, 43n

arithmetic, 24

Arminianism, 28 \& $n$

Arnisaeus, Henningus, $21 \mathrm{n}$

Arrianus, Flavius, 35

art, 41

Arthur, King, 35, 37

Ascham, Roger, 38

Ashmole, Elias, 23n

Askew, Egeon, 34

astrology, 19, 23, 30, 31n

astronomy, 19, 23-24, 26

atomism, 20

Ausonius, 38

\section{B}

Bacon, Francis, 1st baron Verulam, 10, 18, 20, 24

Works, 20

Bacon, Roger, 22
Baconianism, 18, 39

Bale, John, The image of bothe churches, 29n

Barclay, William, 42n

De potestate papae, $42 n$

Baron, Robert, 40n

Baronius, Cardinal Caesar, Annales ecclesiastici, 36

Barrow, Isaac, 24

Bartas, Saluste du: see Saluste du Bartas, Guillaume

Bartholin, Thomas, 25 \& $n$ Anatomia, 25n

Historiarum anatomicarum rariorum, 25n

Basil Valantine, 22

Basle, 28

Bate, George, 35

Elenchus motuum nuperorum, 42

Beck, Cave, 39-40 Universal character, 39-40

Beguin, Jean, 22

Behmenism, 3, 5, 6, 30n, 31

Bellarmine, Robert, 29n

Berkshire, 31n

Beza, Theodore: see Bèze, Theodore de

Bèze, Theodore de, 28

Bible, 12n, 27n, 29 \& n, 30, 32, 38, 39, 41

New Testament, 2, $12 \& \mathrm{n}, 29$

Old Testament, 3, 12, 29

Biblical commentary, 28-30, 31, 39

Biddle, John, 32

Billingsley, Sir Henry, 24

Blundeville, Thomas, 23n

Bodin, Jean, 43

Methodus, 35

Boehme, Jacob, 3, 5, 6, 31, 39

Mysterium magnum, 31

Bohemia, 30n

Boissard, Jacques, 41

Boitet de Frauville, Claude, 42

Bombast von Hohenheim, Philipp Aureol Theophrast: see Paracelsus, Theophrastus

Booker, John, 23n

Borel, Pierre, 24n

Borrichius, Olaus, 17

Hermetis, Aegyptiorum, et chemicorum sapientia, $17 \mathrm{n}$

botany, 19

Boulliau, Ismael, 23

Boyle, Robert, 10n

Bradfield, Berkshire, 31n

Brahe, Tycho, 23, 24n

Brendel, Zacharias, 22

Brightman, Thomas, 29n

Brinsley, John, 39 \& n

Britain, 35

Brugis, Thomas, 26

Brunfels, Otto, 28n

Brutus, 35, 37

Buchanan, George, 38

Rerum scoticarum historia, 35, 42n

Budé, Guillaume, 38

Bullialdus, Ismael: see Boulliau, Ismael

Bunworth, Richard, Man in paradise, 34n

Burgersdijck, Franco, 41

Burggrav, Johann Ernst, 26

Burnet, Duncan, 22

Buteo, Joannes, 24n

Buxtorf the Elder, Johannes, Epitome, 39n 


\section{C}

cabbalism, 19, 23

Caesar, Julius, Commentaries, 35

calculus, differential, 24

Calvin, Jean, 27

Calvinism, 21, 27-29, 33, 34 \& n, 40, 41

Cambridge, University of, $1,18,20 \&$ n, 21, $40 \& n$

Camden, William, 35

Cameron, John, 34n

Myrothecium, 34n

Camfield, Benjamin, 14

Carew, Thomas, 38

Carpenter, Nathanael, 24n

Carrichter, Bartholomaeus, 26

Cartwright, Thomas, 28

Casaubon, Meric, 9 \& n, 17 \& n A true and faithful relation of . . Dr. John Dee, 9n, 26n

Case, John, 43n

Summa, 41

Catholicism, Roman: see Rome, Church of Cauliaco, Guido de: see Guido de Cauliaco

Cebes, 40n

Cervantes, Miguel de, Don Quixote, 38n

Céspedes Y Meneses, Gonzalo de, 37n

Chaldeo-Syriac, 38n

Champaignac, Jean de, 40n

Charenton, Protestant church of, $34 n$

Charles I, King of England, 33, 35

Charles II, King of England, 7

Charleton, Walter, 26

Chaucer, Geoffrey, 38n

Chauliac, Guy de: see Guido de Cauliaco

chemistry, $7 \&$ n, $10 \& n, 18 \& n, 19,22,26$

Chetham's Library, Manchester, 15, 16n

Cheyneius, Jacobus, 2 ln

Chiaramonti, Scipio, De universo, 23

China, 36

chivalry, 37

Christmannus, Jacobus, 23n

Chrysostom, Saint John, 34

church history, 34, 36

Chytraeus, David, 28n

Cicero, 38

circulation of the blood, $25 \& \mathrm{n}$

civil war, English, 1, 2, 30, 30n-31n, 35, 42

Clarke, John, 38n

Clement VIII, Pope, 20n

Clitheroe, 1, 2, 6-7, 10n, 14, 15, 17, 18n, 25, $3 \ln , 33,35,42$

Grammar school, 1, 2n

Coimbra, University of, 20

Comenius, Jan Amos, 21 \& n, 39 \& n Janua linguarum, 39n

Pansophiae prodromus, 21

concordances, biblical, 27n, 29 \& n

congregationalism: see Independency

Conringius, Hermann, 17 \& $n$

De hermetica Egyptiorum vetere, 17n, 26n

Contarini, Cardinal Gasparo, 36n, 42n

Conti, Antonio Maria de', 38n

conveyancing, 42

Copenhagen, 17

Copernicus, Nicolaus, 23

Cornacchinius, Thomas, 25

Corporation Act, 1661, 10n corpuscularianism, $9,10 \& n$

Corser, Rev. Thomas, 15 \& n

cosmography, 19

Costes de la Calprenède, Gautier de, Cassandre, $37 n$

Cotta, John, 26n

Cotton, Sir Robert Bruce, 35

counter-reformation, 36

Coxwold, Yorkshire, 1

Croll, Oswald, 22n, 26, 32n

Cromwell, Oliver, 2, 32

Crossley, James, 15, 16n

cryptography, 40n

Culpeper, Nicholas, 23n

The English physitian, 23n

Curtis, Mark, 18n

Curtius Rufus, Quintus, 35

Cusa, Nicholas of, 18

Daillé, Jean, 28n, $34 \&$ n

Traicté de l'employ des sainct peres, 34

Daneau, Lambert, 21n-22n, 28, 29

Ethices christianae, 40

Daniel, book of, 29 \& $n$

Danzig, 41

Dariot, Claude, 23n

Davenant, Sir William, 38

Gondibert, 37n

Davies, Sir John, 38

Davisson, William, 22

Dee, John, 9n, 24, 26n, 35n

Dell, William, 32, 43

demonology, 2, 3n, 7, 9-13, 17n

demons, devils and evil spirits: see demonology

Demosthenes, 38

D'Eremita, Donato, 22

Derry, 28

Descartes, René, 10, 20, 40 \& n

Opera, 20

Deusingius, Antonius, 25

devil, the (Satan), 2-3, 4n, 9n, 11-13, 32

diabolic possession, $12 \mathrm{n}$

dictionaries, 19, 38-39

Digges, Leonard, 24n

Digges, Thomas, 24n

Diodorus Siculus, 35

Dion Cassius, 35

Dionysius of Halicarnassus, 35

divine right, theory of, $42 \&$ n

divinity: see theology

Downame, George, 28, 41

drama, 37, 38 \& $n$

Drayton, Michael, 38

Du Chesne, Joseph, 22n, 26

Du Jon, Francois, 28

Du Moulin the Elder, Pierre, 28n

Durham, 8

Dutch, 19, $29 \&$ n

\section{$\mathbf{E}$}

Eden, garden of (Paradise), 3, 23

educational reform $2,3,4-6,18 \& \mathrm{n}, 20-24,30$, $37,39,40,41,43$ 
emblem books, 40

encyclopaedia, 18, 21, 23

Endor, witch of, 12

England, 27, 34, 36, 37

Church of, 1, 2, 4 \& n, 5, 7, 9-10, 10n, 27, 32, 33

English, 19, 29 \& n, 31, 36n, 37 \& n, 38 \& n

English-Latin, 38n

Ephesians, 12

Epictetus, 40

Enchiridion, 40n

Epicureanism, 20

episcopalianism, 4

Eramus, Desiderius, 39

Erastus, Thomas, 26n

Erbery, William, 4 \& n, 6, 31 \& n, 32

The testimony, $31 \& \mathrm{n}$

ethics, 21, 40

Euclid, 24n

Geometry, 24

Euripides, 38

Europe, 27, 34, 36, 37

Eustachius, 40n

Eve, temptation of, 12

Everard, John, Some gospel treasures opened, 13n

Everard, Robert, 32

experimentalism, $5,6 \mathrm{n}, 18$

\section{$\mathbf{F}$}

Fabre, Pierre Jean, 22n

Fabricius, Wilhelm, 25

fall of man, 2, 3, 5

familism, 32, 33: see also Grindletonianism

Ranters

Family of Love: see familism

fathers of the church, 27n, 28, 34

Felgenhauer, Paul, 30 \& n

Postilion, 30 \& $n$

Fenner, Dudley, 28, 41

Fernel, Jean, 25

Ficino, Marsilio, 20

fideism, 4, 5, 31-32, 32n

Finckius, Joannes, 26

Finé, Oronce, 24n

Fioravanti, Leonardo, 22n,

Flacius, Matthias, Magdeburg centuries, 36

Florus, Publius Annius, 35

Fludd, Robert, 5, 10, 13n, 20 \& n

Opera, 16, 20n

Foglietta, Uberto, 36n

Fonseca, Petrus, 20

Forestus, Petrus, 25

Franconia, 22n

Freigius, Johann Thomas, $21 \& \mathrm{n}, 40,41$

French, 19, 29, 37, 38

French, John, 17-18, 22

Fuchs, Leonhard, 25

\section{G}

Gaffarel, Jacques, Unheard of curiosities, 23n

Gainsford, Thomas, The historie of Trebizond, 37
Galen, 25 \& n, 26n, 32n, 43

Methodus medendi, 25

Opera, 25

Galenism, 10, 25, 26-27

Galilieo Galilei, 10, 23

Gallucci, Giovanni Paolo, 23n

Gassendi, Pierre, 20

Gellius, Aulus, 38

Genesis, book of, 12, 23, 29, 31

Geneva, 22 \& n

Genoa, 36n

geocentric system, 23 \& n

geography, 19

geometry, 24

German, 19, 22, 29, 38 \& n

Gesner, Conrad, 22

Historia animalium, 16

Gesner, Solomon, 28n

Gilbert, William, 20

Gislenius de Bousbecq, Angerius, 36

Giuntini, Francesco, 23n

glands, 25

Glanvill, Joseph, 9-10, 14, 17n

Glauber, Johann Rudolph, 22 \& n

Glisson, Francis, 25 Anatomia hepatis, 25n

Golius, Theophilus, 40

Gorton, Samuel, 32 \& n Antidote against the common plague, 32 An incorruptible key, 32

grace, free, 4-5, 31

grammar, 19, 35, 38-39

grammar schools, instruction in, $1,2 \mathrm{n}, 35,37$, $38,39 \& n, 41$

Granger, Thomas, 41 Syntagma logicum, 41

Greaves, Richard, 24

Greece, ancient, 34

Greek, 19, 29, 37, 38 \& n, 39 \& n

Greek-Latin, 19, 20, 38 \& n

Grembs, Franciscus Oswald, 26 \& n Arbor integra et ruinosa hominis, 26n

Greville, Robert, $21 \mathrm{n}$

The nature of truth, $21 \mathrm{n}$

Grindleton, Yorkshire, 2, 33n

Grindletonianism, 1, 33

Groot, Hugo de, 2 ln

Grotius, Hugo: see Groot, Hugo de

Gualdo Priorato, Galeazzo, 36

Gualtherus, Rudolphus: see Walther, Rudolph

Guevara, Antonio de, 43

Guez de Balzac, Jean Louis, 42

Guicciardini, Francesco, History, 36n

Guido de Cauliaco, 26

\section{H}

Hakluyt, Richard, 35

Hall, Thomas, 39n

Hartmann, Johann, 22 \& n

Harvard University, $41 \mathrm{n}$

Harvey, William, 10, 25

De motu cordis, 25

heaven, $3,4 \mathrm{n}$

Hebrew, 19, 23, 29 \& n, 38 \& n, 39 \& n

Heidelberg, University of, $29 n$ 


\section{Index}

Heinsius, Daniel, 38n

heliocentric system, 23-24, 24n

Heliodorus, Bishop of Tricca, Ethiopian history, 37

hell, 2-3, 4n

Helmont, John Baptist van, 5, 6n, 10, 13 \& n, $26,32 n$ Opera, 26

Helmontianism, Helmontians, 7, 9, 11, 23n, $26 \& n$

Helmstadt, Universtity of, $21 \mathrm{n}$

Hemmingsen, Niels, 34

Henri IV, King of France, 20n

Herbert, George, 38

Herborn, University of, 21 n, 28

Herigone, Pierre, 24n

hermeticism, 9, 10 \& $n, 12 n, 13,17,19,21-23$, $26,40 \mathrm{n}$

see also: Helmontianism; Paracelsism

Herodian, 35

Herodotus, 35

Hesiod, 38

Heurnius, Joannes, 25

Heydon, Sir Christopher, 23n

Heywood, Thomas, The life of Merlin, 35

Hill, Nicholas, 24n

Hippocratic school, 25

history, 19, 34-36

Hobbes, Thomas, 43

Holinshed, Raphael, 35

Holland, 36

Holwarda, Joannes Phocylides, 20, 24n

Hoppen, K.Theodore, 9n

Hopton, Arthur, 24n

Horace, 38

Hotman, François, 28n, 41, 42

Hues, Robert, 23n

Hugo, Hermannus, 36

Huguenots, $21 \mathrm{n}, 28 \&$ n

humanism, 29n, 38, 39

Huniades, Johannes, 10n

hunting, 18

husbandry, 19

Huygens, Christian, 24n

Hyphantes, Johannes, pseud., 16n

\section{I}

Independency, 4, 7n, 32

Inman, Robert, 30n-31n

Isocrates, 38

Italian, 19, 29 \& n, 36n, 37, 38 \& n

Italian-English, 38n

Italy, 36 \& n

\section{J}

Jacchaeus, Gilbert, $21 \&$ n, 40n

Jack, Gilbert: see Jacchaeus, Gilbert

James I, King of England, 29n

Basilicon doron, 43

Daemonologie, $17 \mathrm{n}$

Jesuits, 20, 21 \& n, 29n, 36

Jobe, Thomas Harmon, 9-10
John Chrysostom, Saint: see: Chrysostom, Saint John

Johnston, Arthur, 38

Jolly, Thomas, $7 \mathrm{n}$

Jonson, Ben, 38n

Juvenal, 38

Junius, Adrianus, 41

Junius, Franciscus: see Du Jon, François

Junius, Melchior, 38n

Justinianus, Petrus, 36n

K

Keckermann, Bartholomaeus, 34, 40n, 41 \& n Opera, 4ln

Systema logica, 41

Keppler, Johann, 23

Khrypffs de Cusa, Nicholas: see Cusa, Nicholas of

Khunrath, Heinrich, 22n

Kildwick, Yorkshire, 1, 8n

Kittredge, G.L., 9n

Knox, John, 29n, 42n

Appelation, 29n

Komenský, Jan Amos: see Comenius, Jan Amos

\section{$\mathbf{L}$}

Lacinius, Janus, 22n

lacteal chyle, 25

Lambert, John, 30n

Lancashire, $1,15,32 \mathrm{n}$

language, universal, 3, 5-6, 23, 39-40, 40n

languages, foreign, 19, $21 \mathrm{n}$ : see also under separate headings for individual languages

Lansbergen, Phillipus van, 24n

La Primaudaye, Pierre de, The French academy, $18,23 n$

La Ramée, Pierre de: see Ramus, Petrus

Latin, 19, 22, 29 \& n, 38 \& n, 39

Latin-French, 38n

Latin-German, 38n

Latin-Italian, 38n

Latin-Teuton, 38n

latitudinarianism, 9

law, 19, 41-42

law, land, 42

laws, English, 42

Lawson, Thomas, 32n

Dagon's fall before the ark, 32n

Lefèvre d'Etaples, Jacques, 40

Le Roy, Louis, 24n

Le Roy, Marin, Polexander, 37n

L'Espine, Jean de, 28n

lexicons: see dictionaries

Leyden, University of, $21 \mathrm{n}$

Leyser, Polycarp, 28n

Lilly, William, 23n

linguistics, 19, 21n, 38-40

Lipsius, Justus, 35

Politicorum, 42n

Lister, Martin, 8, 17, 22n-23n

literature, 19, 36-38

Lithgow, William, 36

liver, the, 25 
Livy, 35

Lodowick, Francis, 39

logic, 6n, 20n, 21, 34, 40-41

Lombard Street, London, 3n, 4

London, 2, 3n, 4, 10n, 17, 42

Low countries, the, 36

Lucanus, Marcus Annaeus, 35

Lucian, 38

Lull, Ramón, 22

Lundorp, Michael Caspar, 36

Lutheranism, 27n, $28 \&$ n, 33, 36

lycanthropy, $12 \mathrm{n}$

Lyly, John, Euphues, 37

lymphatic system, 25 \& n

\section{$\mathbf{M}$}

Machiavelli, Niccolò, 42n, 43

Macropedius, Georgius, 38n

Maestlin, Michael, 24n

magic, natural, 10, 13, 19, 23

Magirus, Joannes, 20 \& n, 40

Magna carta, 42

Magnenus, Joannes Chrysostomus, 20

Maier, Michael, 22

maleficium, 13

manuals, preaching, 34

Manuzio, Paolo, 35

Marischal College, Aberdeen, 21n

Martial, 38

Martini, Jacob, 40n

Martinius, Petrus, 39n

mathematics, 19, 24, 26, 40

Matthew, gospel of, 12

Mazzotta, Benedictus, 23n

mechanical philosophy, 9, 10 \& n

mechanics, 24

medicine, 1, 5, 6n, 7, 9n, 10, 17, 19, 22, 24-27,

30n, 31n, 43

Melanchthon, Philip, 40

Mercator, Gerard, Atlas, 16

Mersenne, Marin, 34n

Mestrezat, Jean, 28

metaphysics, 20 \& n, 21 \& n, 40 \& $n$

meteors, 13n

Metius, Adrian, 23n

Mexico, 20n

millenarianism, 2-3, 6, 7, 23, 29-30

Milton, John, 42

mineralogy, 19, 22n-23n

miracles, 12,13

Mitton, Yorkshire, 2

Montemayor, Jorge de, Diana, 37

Moore, Sir Jonas, 8

More, Henry, 14

Conjectura cabbalistica, 23n

More, Sir Thomas, 38n

Morgan, John, 4

Mornay, Philippe de, 28n

Mueller Regiomontanus, Johann, 24n

mysticism, religious, 31-32

Napier, John, 24n

Neile, Richard, 1
Nelson, Peter, 8 \& $\mathbf{n}$

neo-platonism, 20, 21

Neuhusius, Henricus, 26n

Niclas, Hendrik, 33

\section{O}

occult philosophy: see alchemy; astrology;

Helmontianism; hermeticism; magic, natural;

Paracelsism

Oldenburg, Henry, 8 \& n

optics, 19

Osorio da Fonseca, Jeronimo, 43

Oughtred, William, 24

Clavis mathematica, 24

Ovid, 38

Owen, John, 32-33

Of schisme, 32-33

Oxford, 24

Oxford, University of, 18, 20 \& n, 40

\section{$\mathbf{P}$}

Pacius, Julius, 20, 42

Padua, University of, 20n

Palmendos, 37

Palmerin, 37

pansophism, 21-22

Panvinio, Onofrio, 35

Pappus of Alexandria, 24n

Paracelsians, Paracelsism, 5n, 7, 9, 12n, 17, 20 , $22 \& \mathrm{n}, 26$

Paracelsus, Theophrastus, 5, 10, 13, 22 \& n, $26 \& n$

Opera omnia, 22 \& n

Pare, Ambroise, 25

Pareus, David, 29 \& n, 42n

Parliament, armies of, 1, 26, 31n

Barebones, 2

Cavalier, 7

Parliamentarianism, 42

Paruta, Paolo, 36n, 42n

patristic learning: see fathers of the church

Patrizi, Francesco, Bishop of Gaeta, 42n

Patrizi, Francesco, Platonist, 20, 21

Nova de universis philosophia, 21

Paul, Saint, 12n

Pausanias, 35

Pavone, Francesco, 40

Peccettius, Francescus, 25

Pecquet, Jean, 25

Pererius, Benedictus, 20n

Physica, 20 \& n

perfectionism, 1, 3, 4n

Perkins, William, 28, 41

Petronius, 38

Philippson Sleidanus, Joannes, Commentaries, 36

philosophy, 5, 10,19, 20n, 21, 40-41

philosophy, natural, $3,5-6,7,9-10,12,17$, $19-27,30,36,39,43$

Phioravant, Leonard: see Fioravanti, Leonardo

Pindar, 38

Pinnell, Henry, 5n 


\section{Index}

Piscator of Herborn, Johann, 28, 41

Pistorius, Johann, Artis cabalisticae, 23

Plato, 18

Plautus, 38

Plutarch, 40n

Pocklington, John, Altare christianum, 28

poesy: see poetry

poetry, $37 \& \mathrm{n}, 38,41$

Polanus, Amandus, 28, 41

politics, 19, $29 \& \mathrm{n}, 30,35,41,42-43$

polyglot, 19, 38n

Pomo, Pietro, 36

Pont-à-Mousson, 42n

popery: see Rome, Church of

Pordage, John, 31 \& n

Innocence appearing, 31 \& $\mathrm{n}$

Postel, Guillaume, Absconditorum a

constitutione mundi clavis, 23

Potier, Michael, 22n

Potter, Bertha, In

Powel, Gabriel, 29

pre-destination, 18

presbyterianism, $-4,31 \mathrm{n}-32 \mathrm{n}, 39 \mathrm{n}$

Price, Sir John, Historiae brittanicae defensio, 35

prophecy, 18, 30

prophetic books: see Daniel; Revelation

protestantism, 9, 20, 21, 29, 33-34, 36, 41

Prynne, William, 28

Psalms, book of, 29 \& n

psychology, 19

Ptolemaic system: see geocentric system

Pudsay, Ambrose, 7

puritanism, $1,4,28,30,33,37,38,41$

\section{Q}

Quakers, $32 \&$ n

Quercetanus, Joseph: see Du Chesne, Joseph

Quintana, Francisco de, 37n

Quintilian, 38

$\mathbf{R}$

Rabelais, Francois, 38n

Ramesey, William, 23n

Ramism, 28, 41

Ramus, Petrus, 28n, 41n

Dialectica, 41

Ranters, the, 4n, 31

rationalism: see reason

Ravisius Textor, Joannes, 38n

reason, $4,5,6,11,32 n, 33,34 \& n$

Regiomontanus, Johann: see Mueller Regiomontanus, Johann

Regius, Henricus, 20

Remonstrants, 28

republicanism, 36n, $42 \&$ n

Restoration of 1660 , the, $7,8,9$

Reuchlin, Johann, De verbo mirifico, 23

Revelation, book of, 2, 29 \& n, 30

rhetoric, $20 \mathrm{n}, 37,38$

Rhode Island, 32n

Rhumel, Johann Pharamund, 26

Ridley, Mark, 24n
Riger, Johannes, 40

Ripley, George, 22

Rivet, André, 28n

Robinson, Edmund, 8n

Rollock, Robert, 28

romance, Arthurian, 35, 36-37

Romans, book of, 29 \& $n$

Rome, ancient, 34

Rome, Church of, 13n, 27, 28, 29, \& n, 36, 42n

Rosicrucians, 26n, 30n

royalism, 35,42

Royal Society, 8, 9, 10

Rudbeck the Elder, Olof, 25 \& n

Ruland, Martin, 22n

Rutherford, Samuel, 28

Ruvio, Antonio, 20 \& n

Sacro Bosco, Joannes de, 23n

Sala, Angelo, 26

Sallust, 38

Salmon, Joseph, Antichrist in man, 32

Saluste du Bartas, Guillaume de, 23n

Samuel 1, book of, 12 \& n

Sanders, Richard, 23n

Physiognomie, 23n

Sandys, George, 36

Sarpi, Paolo, 36n

Historie of the Council of Trent, 36n

satire, 38n

Saumaise, Claude de, 42

Saumur, Protestant church of, $34 n$

University of, $28 \mathrm{n}$

Scaliger, Joseph, 38

Scheibler, Christoph, 40n

Scheunemann, Henningus, 26

Schickard, Wilhelm, 24n

scholastic philosophy, 4, 12, 30, 40, 41

Schonaeus, Cornelius, Terentius christianus, 38

Schoonhovius, Florentinus, 41

science, natural: see philosophy, natural

Scot, Reginald, 12n, 13

Scotland, 28, 35

Scribonius, Gulielmus Adolphus, 40 Physica, 22n

Scultetus, Joannes, 25

Sedan, University of, $28 \mathrm{n}$

Seekers, the, 4

Selenus, Gustavus, 40n

Sendivogius, Michael, $A$ new light of alchymie, 17

Seneca, 40

Sennert, Daniel, 25

Opera omnia, 25

Seton, John, 41

Severinus, Petrus, 22n, 26

Shuttleworth, Colonel, 1

Sidney, Sir Philip, Arcadia, 37

Sigonio, Carlo, 36n

Simpson, William, 23n

Slade, Matthew, 28

Sleidanus, Joannes: see Philippson Sleidanus, Joannes

Snell, Willebrod, 24n

Socinianism, 32, 33 
Spain, 20n, 36

Spanish, 19, 36-37, 38 \& n

Spanish-English, 38n

Spanish-French, 38n

Speed, John, 35

Sponde, Henri de, Epitome, 36

Stand, Lancashire, 15

Starkey, George, 26

Stekius, Joannes, 41

Stevin, Simon, 24n

Stow, John, 35

Strada, Famianus, 36

Stuarts, Kings of England, 35, 42

Sturm, Johann, 41

Suetonius, 35

surgery, 1, 25-26, 26n

surveying, 24

syllogism, 34

Syriac, $38 \mathrm{n}$

$\mathbf{T}$

Tacitus, 35

Talaeus, Audomarus, 38n

Rhetorica, $4 \ln$

Talon, Omar: see Talaeus, Audomarus

Tarnovius, Joannes, 28n

Telesio, Bernardino, 20

Terence, 38

theology, 18, 19, 20n, 27-34, 43

theology, scholastic, 4-5, 18, 27, 28, 34

theosophy, 5-6

Thirty Years War, 36

Thompson, James, 26

thoracic duct, 25

Thorndike, Lynn, 20n

Thornton-on-the-Hill, Yorkshire, 1

Thucydides, 35

Tilenus, Daniel, 28n

Titelmann, Franciscus, 21n, 41

tobacco smoking, 18

toleration, religious, 4,31

Toletus, Franciscus, 20 \& n

Tourney, Garfield, 9n

Toxites, Michael, 22n

travel writing, 36

Trevor-Roper, Hugh (Lord Dacre), 43

Tritheim, Johann, 40n

Trogus Pompeius, 35

Troy, 35

Turkey, 36

Turner, Robert, 12n

Twisse, William, 28

Tychonic system: see Brahe, Tycho

Tymme, Thomas, 23n

$\mathbf{U}$

Uffenbach, Peter, 25

Ulstadt, Philipp, 22n

universal language: see language, universal

universities, 4, 6n, $18 \& \mathrm{n}, 20 \& \mathrm{n}, 22,24,30$,

40, 41, 43; see also under separate title

headings
V

Valerius, Cornelius, 23n

Vedelius, Nicolaus, 34

Velcurio, Joannes, $21 n$

Venice, 36 \& $n$

Vernulaeus, Nicolaus, 38n

Veslingius, Joannes, 25

Vicary, Thomas, 26

Vieta, Franciscus, 24n

Virgil, 38

Vives, Joannes Ludovicus, Colloquia, 39

W

Wallis, John, Arithmetica infinitorum, 24

Walther, Rudolph, 28n

Ward, Seth, 18n

weapon-salve, 11

Webbe, Joseph, 39

Webster, Charles, 2n

Webster, Edward, 1

Webster, John

abandonment of religious radicalism, 6-7,

$10 \& n, 32-33,34 n$

on alchemy, $7 \& \mathrm{n}, 18 \& \mathrm{n}, 19,22,22-23,23 \mathrm{n}$

allegorical view of the scriptures, $2-3,11-12$, $12 \mathrm{n}, 13,32$

antinomianism of, 3

Baconianism of, 18

Behmenism of, 3, 5-6, 31, 39

birth, 1 \& $n$

and the civil war, 1, 26n, 42

death, $2,7 \mathrm{n}, 14,26 \mathrm{n}$

eclecticism of, 10,19-20, 27, 34, 43

education, 1, 21

on educational reform, 2, 3, 4-6, 18 \& $n$,

$20-24,30,37,39,40,41,43$

and William Erbery, 4, 31 \& $n$

and John Everard, 13n

fideism of $4,5,31,32 n$

and Grindletonianism, 1 \& n, 33n

and Thomas Hall, 39n

and hermeticism, 5, 7, 9-10, 10n, 11, 13 \& n,

$17 \& \mathrm{n}, 19,20$ \& n, 22-23

on human learning in religion, 3-6, 30,

31-32, 34

and Robert Inman, 30n-31n

and John Lambert, 30n

and Thomas Lawson, 32n

library of, 15-43

and Martin Lister, 8, 17, 22n-23n

magistrate for Clitheroe, 6-7, 42

and medicine, $1,7,10,19,24-25$

millenarianism of, 2-3, 6, 7, 29-30

minister of the Church of England, 1,

2 \& $n, 8 n$

property owner in Clitheroe, 6,42

and the Quakers, 32n

on human reason, $4,5,6,11,32 \mathrm{n}, 33,34 \mathrm{n}$

religion of, $1,2-7,9-10,10 \mathrm{n}, 11,17,18$,

27-34

and the Royal Society, 8-9, 10

school-teacher, $1,35,37,39 \&$ n

on science, 7, 9-11, 17, 19-27, 39

and William Simpson, 23n 


\section{Index}

surgeon, 1, 26n

will of, $14,15,26 n, 33 n$

on witchcraft, $3 \mathrm{n}, 7-14,19,33$

Weigel, Valentine, 31

of the life of Christ, 31

Wharton, Thomas, 25

Whitechapel, London, 10n

Wier, Johann, De praestigiis daemonum, 17n

Wilke, Andreas, 38n

Wilkins, John, 18n, 23, 39

Willet, Andrew, 29

Williams, John, 28

Willis, Thomas, 10n

wills, 42

Wishart, George, 35

witchcraft, 3n, 7-14, 17n, 19, 32, 33

Witekind, Hermann, 23n

Wither, George, 34, 38n

Wittenberg, University of, 30n

Wood, Anthony, 1, 21

Woodall, John, 25

Woodcock, Francis, 30

Worm, Olaus, Museum, 23

Wuertz, Felix, 26

Xenophon, 35

$\mathbf{Y}$

York, 23n

Yorkshire, 1, 2n, 8, 33n

Zabarella, Giacomo, 20 \& n

Zasius, Ulrich, 42

Zetzner, Lazarus, 22n

zoology, 19

Zwinglianism, 28n 